\title{
Mapping QTLs for Sheath Blight Resistance in the Rice Line WSS2
}

\author{
Hiroyuki Sato*1), Osamu Ideta'), Ikuo Ando'), Yasufumi Kunihiro'), Hideyuki Hirabayashi'), \\ Masataka Iwano ${ }^{3)}$, Atsushi Miyasaka ${ }^{4}$, Hiroshi Nemoto ${ }^{1)}$ and Tokio Imbe ${ }^{1)}$ \\ 1) National Institute of Crop Science, 2-1-18 Kannondai, Tsukuba, Ibaraki 305-8518, Japan \\ 2) National Agricultural Research Center for Hokkaido Region, 1 Hitsujigaoka, Toyohira, Sapporo, Hokkaido 062-8555, Japan \\ 3) National Agriculture Research Center, 1-2-1 Inada, Joetsu, Niigata 943-0193, Japan \\ 4) National Agricultural Research Center, 3-1-1 Kannondai, Tsukuba, Ibaraki 305-8666, Japan
}

The rice line WSS2 which was derived from the Vietnamese indica variety Tetep, displays a high partial resistance to sheath blight. Quantitative trait locus (QTL) analysis of the resistance using simple sequence repeat (SSR) and sequence-tagged site (STS) markers was conducted in a $\mathrm{BC}_{1} \mathrm{~F}_{1}$ population derived from the cross Hinohikari/WSS2//Hinohikari. Sheath blight resistance in this population and its cross-parents was studied using syringe inoculation. Two QTLs for sheath blight resistance ( $q S B-3$ and $q S B-12)$ were identified on chromosomes 3 and 12. Their resistance alleles were derived from the resistant parent WSS2. These QTLs totally explained $29.6 \%$ of the phenotypic variation. Sheath blight resistance was significantly correlated with culm length and heading date. Among the QTLs for culm length and heading date, $q C L-3$ for culm length was located in the same region as $q S B-3$, and the remaining QTLs were not linked to $q S B-12$. Thus, it was reasonable to assume that $q S B-12$ would enable to breed a rice variety resistant to sheath blight.

Key Words: Oryza sativa L., sheath blight, partial resistance, SSR marker, STS marker, QTL analysis.

\section{Introduction}

Sheath blight, caused by the pathogenic fungus Rhizoctonia solani, is the second most prevalent disease in rice after blast disease in Japan (Hori 1991). The epidemic area for sheath blight extends over 1 million ha (Hori 1991) and yield loss caused by it is the most serious in warm regions of Japan (Oohata 1989). Despite the commercial importance of improving rice resistance to this disease, genetic studies have progressed more slowly than expected (Pan et al. 1999) because the pathogen is saprophytic and parasitic with a wide host range. The association between disease development and several morphological plant properties, such as culm length and heading date, has been frequently observed (Hori

Communicated by H. Ikehashi

Received January 26, 2004. Accepted May 7, 2004.

*Corresponding author (e-mail: bsato@affrc.go.jp)
1991). In the last 2 decades, transgenic rice lines resistant to sheath blight have been developed (Datta et al. 2000, Zhang et al. 2001) and significant differences in resistance among rice varieties and lines were confirmed (Pan et al. 1999, Wasano et al. 1983, Groth and Nowick 1992, Li et al. 1995, Zou et al. 2000, Kunihiro et al. 2002, Han et al. 2002, Xie et al. 1990). The quantitative resistance in one of the most resistant varieties, Tetep offers excellent protection against the pathogen under field conditions (Wasano et al. 1983, Groth and Nowick 1992) and this variety shows fewer and smaller lesions, suggesting the presence of physiological mechanisms of resistance (Groth and Nowick 1992). Wasano (1988) reported that the resistance in Tetep was controlled by one or 2 dominant gene(s). However, the chromosomal location and tightly linked markers of the resistance gene(s) have yet to be identified. Wasano (1988) developed a shortculm elite line, 2F15-92-9-22-20-11-29 (WSS2), from the progeny of Tetep. Although the tendency toward lodging in Tetep had been improved in WSS2, the other agronomic characteristics, especially fertility and yield, still remain extremely poor. Therefore, when breeders use the source of resistance, it is necessary to conduct continued backcrossing to cultivars with good agronomic characteristics. We aimed to introduce the sheath blight resistance from WSS2 to Hinohikari, which is a leading variety in warm regions of Japan where the disease is most serious. The objective of this study was to identify molecular markers tightly linked to sheath blight resistance gene(s) in WSS2 to facilitate markerassisted selection in the continued backcrossing program and to map gene(s) on rice chromosomes to provide detailed information about resistance loci for future investigations.

\section{Materials and Methods}

\section{Plant materials}

Seeds of the sheath blight resistant line 2F15-92-9-2220-11-29 (WSS2) were supplied by Dr. Kikuo Wasano (Saga University). WSS2 is the progeny of a hybrid population derived from the cross CN4-4-2 and a resistant Vietnamese variety, Tetep (Wasano 1988). A cross between WSS2 and a disease-susceptible Japanese variety, Hinohikari, was conducted in the summer of 1999, and the resulting $F_{1}$ plants were backcrossed to Hinohikari in 2000. The $60 \mathrm{BC}_{1} \mathrm{~F}_{1}$ plants developed from the crosses, Hinohikari/ WSS2//Hinohikari, and the cross-parents ( 5 plants for each 
parent) were grown at the Yawara Lowland Experimental Station of the National Institute of Crop Science, Japan, in 2001. Seeds were sown on April 17 and 30-day-old seedlings were transplanted at the rate of 1 plant per hill. The plot consisted of 3 rows $10 \mathrm{~m}$ long with $60 \mathrm{~cm}$ spacing between rows. Hill space within rows was $30 \mathrm{~cm}$. Basal application of chemical fertilizer was performed at the rates of $88 \mathrm{~kg} \mathrm{~N}$, $88 \mathrm{~kg} \mathrm{P}$ and $66 \mathrm{~kg} \mathrm{~K} \mathrm{ha}^{-1}$ just before transplanting. Heading date, culm length and sheath blight resistance were evaluated for each individual.

\section{Evaluation of sheath blight resistance}

The Rhizoctonia solani isolate CS-2 maintained in the Fungal Disease Lab. of the National Agricultural Research Center was used for inoculation. Culture and inoculation of the mycelium were based on the syringe inoculation method (Wasano et al. 1983) with the following modifications: To maintain the fungal virulence, $1 \%(\mathrm{w} / \mathrm{v})$ polypeptone was added to Potato Sucrose Agar (PSA) medium containing 200 $\mathrm{g}$ of potato, $20 \mathrm{~g}$ of sucrose, and $10 \mathrm{~g}$ of agar per $\mathrm{L}$ in volume. To speed up inoculum preparation, the mycelium in the PSA medium was homogenized with a food processor (Food Millser IFM-15, Iwatani) and $0.2 \mathrm{ml}$ of the prepared inoculum was injected to the third leaf sheath from the flag leaf using a plastic syringe at the heading date. Disease symptoms were scored based on the ratio of lesion area to total leaf sheath area of the second leaf 28 days after inoculation, according to the method of Wasano et al. (1983). Three leaf sheaths per plant were used for inoculation. The average disease score of the 3 replications per plant was then used to express the degree of resistance of the plant in QTL analysis.

\section{Development of new SSR markers}

Three new SSR markers -- J1202, J1205 and J1206 -were developed for chromosome 12. J1202 was developed on a rice $\mathrm{BAC}$ clone, $\mathrm{BX} 000510$, and the remaining 2 markers on another rice BAC clone, OSJNBb0059G18. SSR regions on the BAC clones were searched for, using the SSRIT program (Temnykh et al. 2001). Primer pairs for each marker were designed by using HYB simulator software ver. 4 (Advanced Gene Computing Technology). Nucleotide sequences of the primer pairs were as follows: J1202, 5'-AGC AGAGGATGGAGAAGTTTCCTAAAGC-3' and 5'-CCAG ACAGAATCTC AGCAAATCAGTGAC-3'; J1205, 5'-CTT GACCTACTTTTAGCAACTTTTCGTAGCC-3' and 5'-TG AACCGGCCCTGAACTTGTAC-3'; J1206, 5'-GGCAATC ACCTGTAA CATTTCGTTCTCT-3' and 5'-AGCAGGTTA GTTCACTCCATGTGCT-3'.

\section{SSR and STS analysis}

Total DNA was extracted from the leaf blades of each plant based on the CTAB method (Murray and Thompson 1980). The PCR reaction for the SSR markers was performed in a $4 \mu \mathrm{l}$ reaction buffer containing 1.25 units of Taq DNA polymerase (Promega, Madison,WI, USA), $0.2 \mathrm{mM}$ of dNTP, $1.5 \mathrm{mM}$ of $\mathrm{MgCl}_{2}, 20 \mathrm{ng}$ of DNA and $20 \mathrm{pmol}$ of each primer. A 384-well thermal cycler (PTC-225, MJ Research) was used for the reaction. PCR cycling for SSR was described by Kono et al. (2000). To detect polymorphism, the amplified product was separated on $2.5 \%$ to $4 \%$ MetaPhor agarose gel (BMA Inc., Rockland, Maine, USA) at $200 \mathrm{~V}$ for $100 \mathrm{~min}$. Primers for STS markers were synthesized according to the published primer sequences by the Rice Genome Project, Japan. The PCR conditions used for STS analysis were based on the method of Wu et al. (2002). The detection of polymorphism was the same as that for SSR analysis.

\section{Map construction and QTL analysis}

A total of 206 SSR and STS markers distributed on the 12 chromosomes were used to construct a rice linkage map with MAPMAKER/EXP version 3.0 (Lander et al. 1987, Lincoln et al. 1993). To avoid detecting pseudo-linkage of markers, a relatively higher threshold level (LOD > 3.0) was employed to construct the linkage map. Map units in $\mathrm{cM}$ were calculated using the Kosambi function. Chromosomal locations of QTLs were determined by single-point analysis using QGene software version 3.06v (Nelson 1997). Regression ANOVA was conducted to test the significance of association at each locus between 2 genotype groups (homozygous for Hinohikari alleles and heterozygous alleles) for 3 traits, i.e., disease score, culm length and heading date. A probability of 0.01 was used as a threshold to detect significant mean differences between the 2 genotype groups for the three traits. Putative QTLs were visualized by using the QGene "multi plot" command. For each trait, the total phenotypic variation explained by all the QTLs was estimated from the QGene "multiple regression" command.

\section{Results}

\section{Linkage map construction}

We initially screened 284 markers that showed polymorphism for WSS2 and Hinohikari among the 862 SSR and STS markers. The frequency of polymorphism was 32.9 $\%$. Using both 201 SSR markers and 5 STS markers (C51416, C53358, C60465, C60248 and C60772), we constructed a linkage map based on $60 \mathrm{BC}_{1} \mathrm{~F}_{1}$ individuals derived from the cross between WSS2 and Hinohikari (Fig. 1). These markers were grouped into 19 linkage groups. Gaps were observed in chromosomes 2, 4, 5, 6, 7, 11 and 12 . The total map distance was $1167.7 \mathrm{cM}$, corresponding to $76.0 \%$ of the published rice genetic map constructed using SSR markers (McCouch et al. 2002). The marker sequence generally agreed with that of previous rice maps (Temnykh et al. 2000, Temnykh et al. 2001, McCouch et al. 2002). The only discrepancy between our map and those of McCouch et al. (2002) involved chromosome 4 (markers RM2441 and RM241). The average distance between the markers was 9.3 $\mathrm{cM}$. Distances between the markers were generally in agreement with those in previously published maps, with slight differences due most likely to the small population (60 individuals). 
Segregation ratios of the 2 genotype classes for most loci fitted the expected ratio of 1 (Hinohikari) : 1 (WSS2). Significant segregation distortions were observed for chromosomes 3, 6 and 8 , at $\mathrm{P}<0.01$ (Fig. 1). The frequency of Hinohikari alleles contributed to increase in the regions on chromosomes 3 and 8, while the skewed region on chromosome 6 revealed on increase in the WSS2 alleles.
Variations in sheath blight resistance, culm length and heading date in the $\mathrm{BC}_{1} \mathrm{~F}_{1}$ population

A significant difference in sheath blight resistance was found between WSS2 and Hinohikari (Fig. 2). The average disease scores for the 2 cross-parents were 33.8 in WSS2 and 66.2 in Hinohikari (Fig. 3A). The disease scores of the $\mathrm{BC}_{1} \mathrm{~F}_{1}$ population ranged from 10 to 80 with continuous variation (Fig. 3A). The culm length of WSS2 was 92.8 \pm 3.8 $\mathrm{cm}$ and that of Hinohikari was $91.3 \pm 3.1 \mathrm{~cm}$. Phenotypic frequency distribution of the $\mathrm{BC}_{1} \mathrm{~F}_{1}$ population for culm

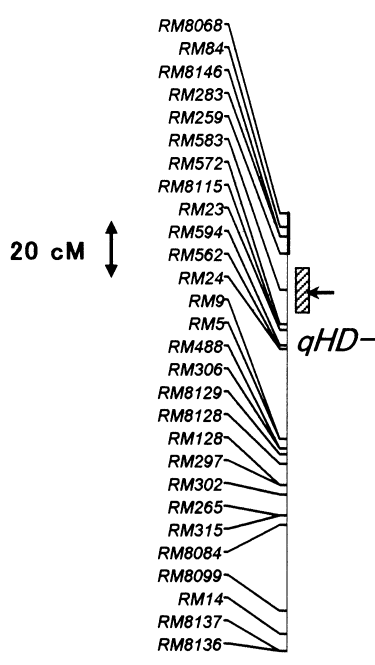

Chr. 1

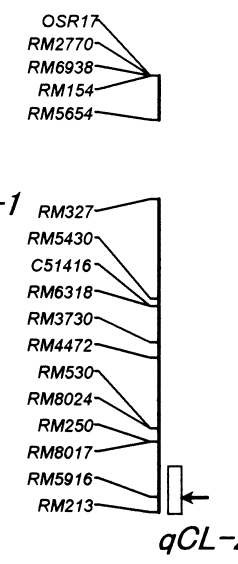

Chr. 2

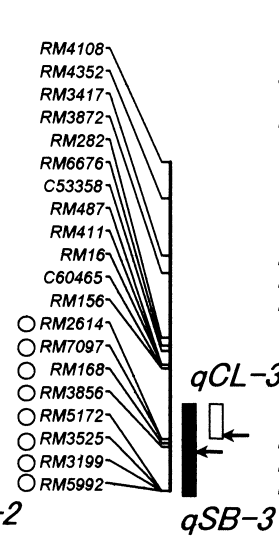

Chr. 3

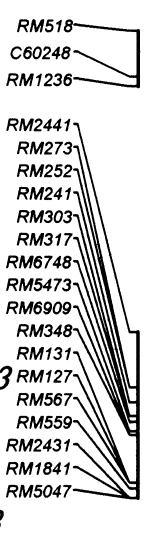

Chr. 4
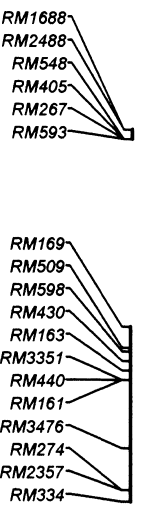

Chr. 5
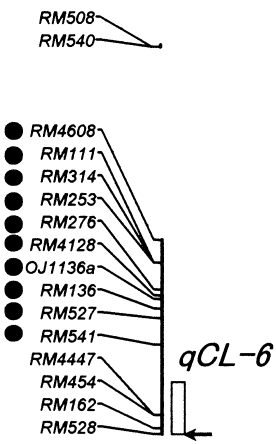

Chr. 6

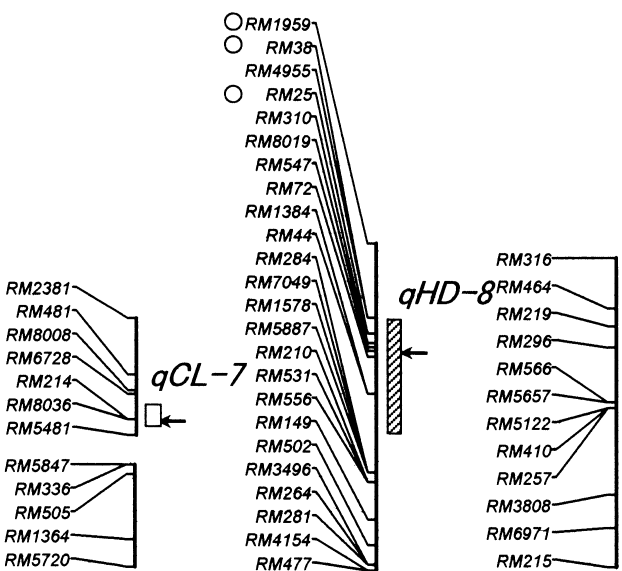

Chr. 7
Chr. 8

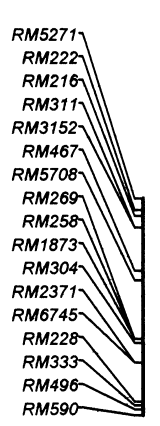

Chr. 10
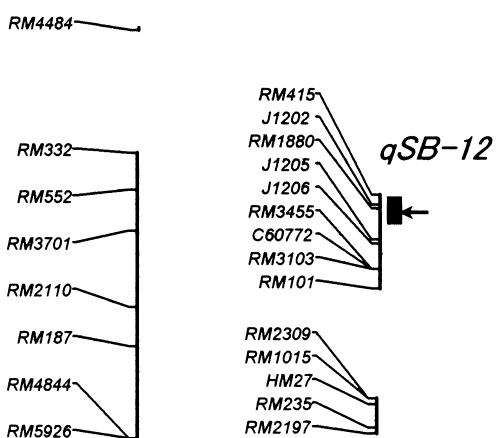

Chr. 11
Chr. 12

Fig. 1. Linkage map and QTL positions. Gray, white and striped bars represent putative regions of QTLs for disease score, culm length and heading date. QTL positions were visualized using the QGene "multi plot" based on ANOVA $(\mathrm{p}<0.01)$. Arrows indicate the nearest marker locus of each putative QTL. Loci with significant segregation distortion from the anticipated segregation ratio $(\mathrm{p}<0.01)$ are denoted by closed $(\mathbf{O})$ or open $(\bigcirc)$ circles. These circles show that the frequency of the WSS2 allele or Hinohikari allele increased in the region. 


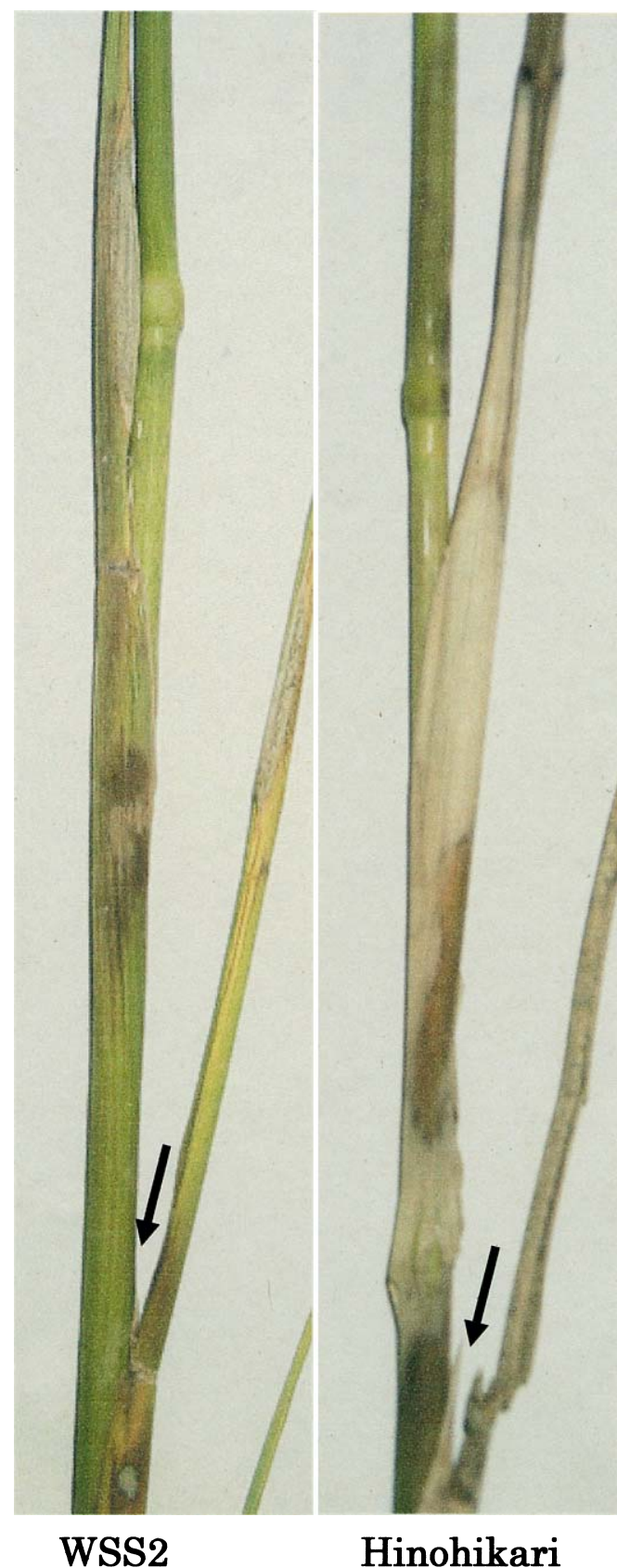

Fig. 2. Disease symptoms on the second leaf sheath from the flag leaf in WSS2 and Hinohikari. Sheath blight was inoculated on the third leaf sheath (syringe inoculation). Arrows show the inoculation site. These pictures were taken 28 days after inoculation. Disease scores for WSS2 and Hinohikari were 33.2 and 71.6.

length varied continuously with transgressive segregants (Fig. 3B). A significant negative correlation was found between the disease score and culm length $(P=0.003)$ (Fig. 4). The days to heading of WSS2 and Hinohikari were 126 and 135 , respectively and segregation in the trait showed a continuous distribution (Fig. 3C). Significant negative correlation $(\mathrm{r}=-0.380, \mathrm{P}<0.01)$ was also evident between the disease score and days to heading, respectively.
A

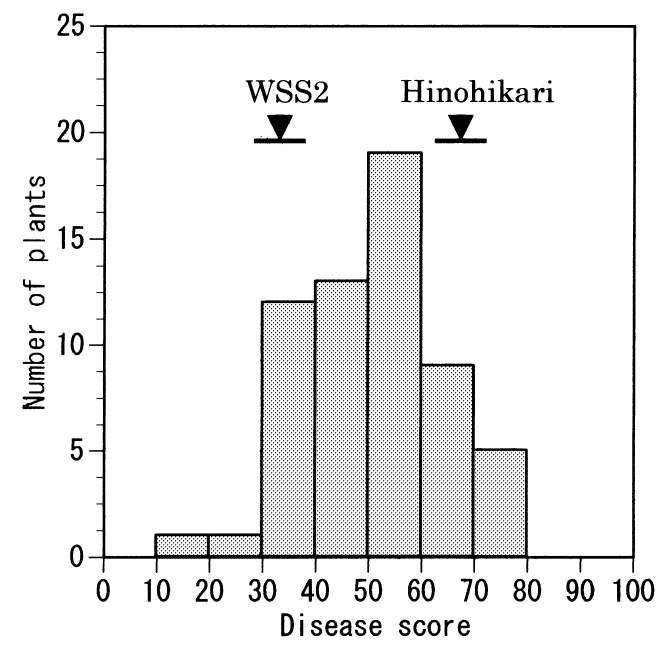

B

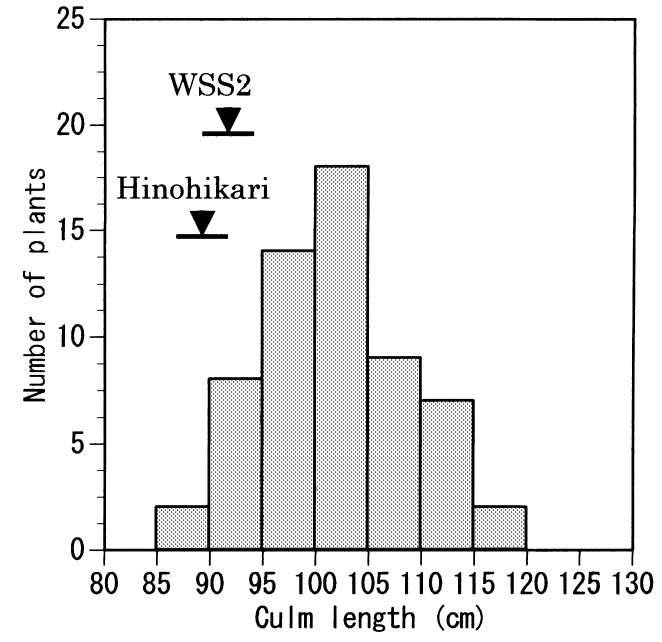

C

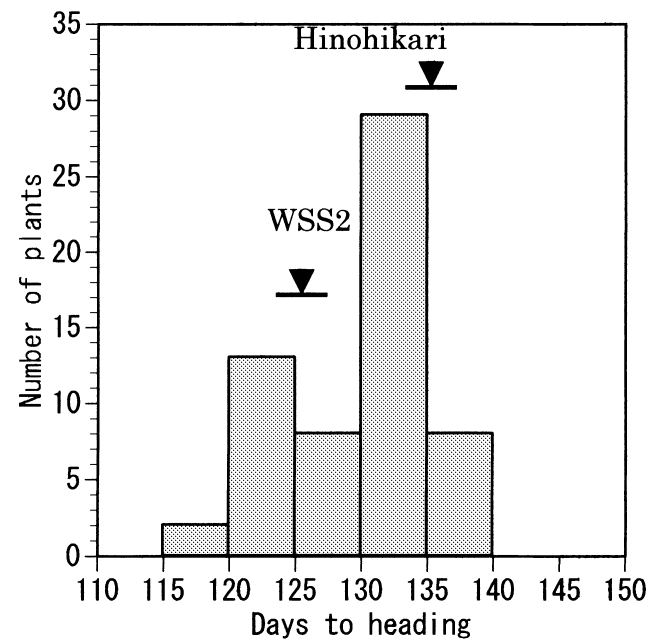

Fig. 3. Frequency distribution for disease score, culm length and days to heading in the $\mathrm{BC}_{1} \mathrm{~F}_{1}$ population of Hinohikari/WSS2// Hinohikari cross. A, disease score; $\mathrm{B}$, culm length; $\mathrm{C}$, days to heading.

\section{QTLs for sheath blight resistance}

Two significant $(\mathrm{P}<0.01)$ QTLs $(q S B-3$ and $q S B-12)$ associated with sheath blight resistance were detected on 


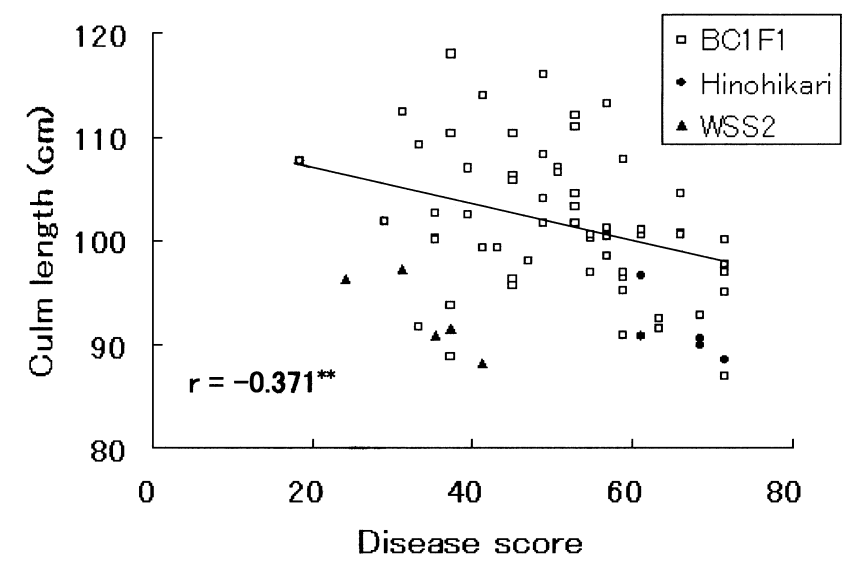

Fig. 4. Relationship between the disease score and culm length. $* *$ : significant at $1 \%$ level

chromosomes 3 and 12 (Fig. 1 and Table 1). Heterozygously, the WSS2 alleles around the SSR markers RM3856 (chromosome 3) and RM 1880 (chromosome 12) decreased the disease score by 14 and 9 , respectively. The percentages of phenotypic variation explained by each QTL were $19.4 \%$ and $12.9 \%$. The percentage of phenotypic variation explained by the 2 QTLs was $29.6 \%$ to the total variance based on multiple-regression analysis. No significant interaction was observed in the 2 QTLs.

\section{QTLs for culm length and heading date}

Significant $(\mathrm{P}<0.01)$ QTLs for culm length $(q C L-2$, $q C L-3, q C L-6$ and $q C L-7)$ were detected on chromosomes 2 , 3, 6 and 7 (Fig. 1 and Table 1). Heterozygously, the WSS2 alleles around the SSR markers, RM7097 and RM2614 (chromosome 3), RM4447 (chromosome 6), and RM8036 (chromosome 7) contributed to the increase of the culm length by $5.6,6.9$ and $4.9 \mathrm{~cm}$, respectively, while another WSS2 allele around RM5916 (chromosome 2) contributed to the decrease of the culm length by $5.2 \mathrm{~cm}$. The phenotypic variation explained by each QTL ranged from $11.0 \%$ to 24.0 $\%$. Based on multiple-regression analysis, these 4 QTLs explained $43.2 \%$ of the total phenotypic variation. No significant interactions were found among QTLs identified.

Two putative QTLs for heading date ( $q H D-1$ and $q H D$ 8) were also identified (Fig. 1 and Table 1). These QTLs were located near RM583 (chromosome 1) and RM547 (chromosome 8). WSS2 alleles around RM583 and RM547 delayed heading by 3.2 and 4.3 days, respectively. Percentages of phenotypic variation explained by these QTLs were $11.9 \%$ and $19.6 \%$, and the cumulative effect was $28.1 \%$. No significant interactions were found among QTLs identified.

\section{Discussion}

Despite several attempts made by rice researchers, no gene conferring true resistance to the sheath blight fungus has yet been identified ( $\mathrm{Li}$ et al. 1995). However, some resistant varieties and lines, i.e., Tetep, Jasmin 85, Teqing, Zhai Ye Qing 8 (ZYQ8), Minghui 63, LSBR-5 and LSBR-33, offer sufficient partial resistance to the pathogen under field conditions to be agriculturally useful (Wasano et al. 1983, Pan et al. 1999, Li et al. 1995, Zou et al. 2000, Kunihiro et al. 2002, Han et al. 2002, Xie et al. 1990). In the present paper, we conducted a QTL analysis for sheath blight resistance in WSS2 derived from Tetep.

We constructed a linkage map based on the $60 \mathrm{BC}_{1} \mathrm{~F}_{1}$ individuals derived from the cross between WSS2 and Hinohikari (Fig. 1). Segregation distortions were observed for

Table 1. Putative QTLs for disease score, culm length and heading date

\begin{tabular}{|c|c|c|c|c|c|c|c|c|c|c|}
\hline \multirow{2}{*}{ Trait } & \multirow{2}{*}{ QTL $^{1)}$} & \multirow{2}{*}{$\begin{array}{c}\text { Chromosome } \\
\text { No. }\end{array}$} & \multirow{2}{*}{$\mathrm{NML}^{2)}$} & \multicolumn{2}{|c|}{ (Phenotypic mean value ${ }^{3)}$} & \multirow{2}{*}{$\mathrm{F}$} & \multirow{2}{*}{$\mathrm{P}$} & \multirow{2}{*}{ LOD } & \multirow{2}{*}{$\%$ Variation $^{4)}$} & \multirow{2}{*}{$\mathrm{DPE}^{6)}$} \\
\hline & & & & $\mathrm{H}(\mathrm{n})$ & W (n) & & & & & \\
\hline Disease & $q S B-3$ & 3 & RM3856 & $53.5(49)$ & $39.5(11)$ & 14.0 & 0.0004 & 2.8 & 19.4 & $\mathrm{H}$ \\
\hline \multirow[t]{2}{*}{ score } & $q S B-12$ & 12 & RM1880 & $54.6(36)$ & $45.6(24)$ & 8.6 & 0.0048 & 1.8 & 12.9 & $\mathrm{H}$ \\
\hline & & & & & & & & Total $^{5)}$ & 29.6 & \\
\hline Culm & $q C L-2$ & 2 & RM5916 & $104.3(29)$ & $99.1(31)$ & 9.8 & 0.0027 & 2.0 & 14.4 & $\mathrm{H}$ \\
\hline \multirow[t]{4}{*}{ length } & $q C L-3$ & 3 & RM7097, RM2614 & $100.4(47)$ & $106.0(13)$ & 7.2 & 0.0095 & 1.5 & 11.0 & W \\
\hline & $q C L-6$ & 6 & RM4447 & $97.6(24)$ & $104.5(35)$ & 17.5 & 0.0001 & 3.4 & 24.0 & W \\
\hline & $q C L-7$ & 7 & RM8036 & $99.2(30)$ & $104.1(30)$ & 8.4 & 0.0052 & 1.8 & 12.6 & W \\
\hline & & & & & & & & Total $^{5)}$ & 43.2 & \\
\hline Heading & $q H D-1$ & 1 & RM583 & $129.2(21)$ & $132.4(37)$ & 7.6 & 0.0078 & 1.6 & 11.9 & $\mathrm{~W}$ \\
\hline \multirow[t]{2}{*}{ date } & $q H D-8$ & 8 & RM547 & $129.4(36)$ & $133.7(21)$ & 13.4 & 0.0006 & 2.7 & 19.6 & W \\
\hline & & & & & & & & Total $^{5)}$ & 28.1 & \\
\hline
\end{tabular}

\footnotetext{
1) Trait abbreviation plus chromosome number horboring QTL.

2) Nearest marker locus of putative QTLs.

3) Phenotypic mean value of individuals carrying the Hinohikari (H) or WSS2 (W) allele. Data in parenthesis indicate the number of plants.

4) Phenotypic variation explained by each QTL.

5) Estimates from the multiple regression command of the QGene software.

6) Direction of phenotypic effect. H and W indicate Hinohikari and WSS2 alleles, respectively which increased the phenotypic mean value.
} 
chromosomes 3, 6 and 8. The distortion in the middle region of the short arm of chromosome 6 may correspond to the distortion toward Indica alleles described by Fukuta et al. (2000), Ishikawa (1994) and Harushima et al. (1996). Based on the location of the markers and skewness, the remaining distortions in our study did not correspond to those reported previously.

Two QTLs, $q S B-3$ and $q S B-12$, were detected for sheath blight resistance and the proportions of phenotypic variation explained by $q S B-3$ and $q S B-12$ were $19.4 \%$ and $12.9 \%$, respectively (Fig. 1 and Table 1 ). The WSS2 allele at QTLs reduced the disease score, as anticipated based on the WSS2 phenotype (Table 1). Wasano (1988) reported that Tetep harbored one or two dominant resistance gene(s). As for the number of resistance genes, our results may be in agreement with the conclusion presented by Wasano (1988). However, there is no conclusive evidence that the two QTLs corresponded to the previously reported gene(s), because both $q S B-3$ and $q S B-12$ were totally explained only $29.6 \%$ of the phenotypic variation (Table 1 ).

It is generally recognized that partial resistance to sheath blight in rice is generally influenced by morphological traits such as culm length and heading date (Hori 1991, Wasano et al. 1983, Li et al. 1995, Zou et al. 2000, Kunihiro et al. 2002). In our data, significant negative correlations were also observed between the disease score and these 2 traits. Negative correlation between the disease score and culm length was particularly high, because two cross parents, WSS2 and Hinohikari, per se showed that in the individuals with a long culm the disease score tended to decrease (Fig. 4). The association between the decreased resistance and increased culm length was explained by the mode of infection of the pathogen, that is, the infection began in the lower leaf sheath and progressed upward (Hori 1991, Wasano et al. 1983). On the other hand, it was assumed that late-maturing plants could avoid the attack by the pathogen because of the lower temperature at heading stage which is the most susceptible stage to the disease (Wasano et al. 1983). As culm length and heading date significantly affected the evaluation of the resistance, we examined the relationships between the resistance QTLs and the genetic regions of the two characters.

The $q S B-3$ QTL was mapped approximately at the same chromosomal location as the QTL for culm length, $q C L-3$ (Fig. 1). Kunihiro et al. (2002) also identified $q S B R-3$ and $q C L-3$ for sheath blight resistance and culm length, respectively, in the same marker interval in a DH population derived from ZYQ8/Jing Xi 17. These QTLs were located at almost the same position as that of ours. They concluded that the effect of $q S B R-3$ may be due to the pleiotropic effect of $q C L-3$ (Kunihiro et al. 2002). Thus, since it was reasonable to assume that $q S B-3$ was affected by $q C L-3$, it is likely that $q S B-3$ will not be useful for the breeding of resistant varieties to the disease.

On the other hand, the other QTL for resistance to sheath blight, $q S B-12$, did not correspond to the QTLs for culm length or heading date (Fig. 1). Therefore, it was assumed that this QTL would be agronomically important to enhance the resistance to the disease. Li et al. (1995) identified 6 QTLs for sheath blight resistance in a $F_{4}$ population of Lemont/Teqin, and one (QSbr12a) was located on chromosome 12. QSbr12a was, however, closely associated with a faint QTL for culm length (Li et al. 1995). The discrepancy was explained by the fact that the position of $Q S b r 12 a$ was more than $30 \mathrm{cM}$ away from that of $q S B-12$ in our study. In addition, no QTL for culm length were identified on chromosome 12 in our mapping population (Fig. 1 and Table 1).

In the present study, we identified 2 QTLs for sheath blight resistance in WSS2. One of the 2 QTLs, qSB-12, could be useful for the enhancement of resistance to the disease, as stated above. In further studies, we could use the SSR marker RM1880 linked to $q S B-12$ to promote the breeding process of rice sheath blight resistance.

\section{Acknowledgments}

We thank Drs. M. Yano and I. Kono of NIAS for their assistance in the SSR analysis. We also thank Prof. Emeritus $\mathrm{K}$. Wasano of Saga University for providing the rice line WSS2. We thank Dr. E.A. Barlaan (Nagasaki Industrial Promotion Foundation) for revising the manuscript.

\section{Literature Cited}

Datta,K., Z.Koukolikova-Nicola, N.Baisakh, N.Oliva and S.K.Datta (2000) Agrobacterium-mediated engineering for sheath blight resistance of indica rice cultivars from different ecosystems. Theor. Appl. Genet. 100: 832-839.

Fukuta, Y., H.Sasahara, K. Tamura and T.Fukuyama (2000) RFLP linkage map included the information of segregation distortion in a wide-cross population between Indica and Japonica rice (Oryza sativa L.). Breed. Sci. 50: 65-72.

Groth,D.E. and E.M.Nowick (1992) Selection for resistance to rice sheath blight through number of infection cushions and lesion type. Plant Dis. 76: 721-723.

Han, Y.P., Y.Z.Xing, Z.X.Chen, S.L.Gu, X.B.Pan, X.L.Chen and Q.F.Zhang (2002) Mapping QTLs for horizontal resistance to sheath blight in an elite rice restorer line, Minghui 63. Acta Genetica Sinica 29: 622-626.

Harushima, Y., N.Kurata, M.Yano, Y.Nagamura, T.Sasaki, Y.Minobe and M.Nakagahra (1996) Detection of segregation distortion in an Indica-Japonica rice cross using a high-resolution molecular map. Theor. Appl. Genet. 92: 145-150.

Hori,M. (1991) Epidemiology and control of rice sheath blight in Japan. Japan Plant Protection Association, Tokyo. 324 p.

Ishikawa, R. (1994) Genetic studies on isozyme genes in rice. Bull. Fac. Agric. Hirosaki Univ. 57: 105-180.

Kono,I., Y.Takeuchi, T.Shimano, T.Sasaki and M.Yano (2000) Comparison of efficiency of detecting polymorphism among japonica varieties in rice using RFLP, RAPD, AFLP and SSR Markers. Breed. Res. 2: 197-203.

Kunihiro, Y., Q.Qian, H.Sato, S.Teng, D.L.Zeng, K.Fujimoto and L.H.Zhu (2002) QTL analysis of sheath blight resistance in rice (Oryza sativa L.). Acta Genetica Sinica 29: 50-55.

Lander, E.S., P. Green, J.Abrahamson, A. Barlow, M.J.Daly, S.E. 
Lincoln and T.Etoh (1987) MAPMAKER: an interactive computer package for constructing primary genetic linkage maps of experimental and natural populations. Genomics 1: 174-181.

Li,Z., S.R.M.Pinson, M.A.Marchetti, J.W.Stansel and W.D.Park (1995) Characterization of quantitative trait loci (QTLs) in cultivated rice contributing to field resistance to sheath blight (Rhizoctonia solani). Theor. Appl. Genet. 91: 382-388.

Lincoln, S.E., M.J.Daly and E.S.Lander (1993) Constructing genetic linkage maps with Mapmaker/EXP version 3.0: a tutorial and reference manual, 3rd ed. Whitehead Institute for Bio-metrical Research, Cambridge, Massachusetts. $47 \mathrm{p}$.

McCouch,S.R., L.Teytelman, Y.Xu, K.B.Lobos, K.Clare, M.Walton, B.Fu, R.Maghirang, Z.Li, Y.Xing, Q.Zhang, I.Kono, M.Yano, R.Fjellstrom, G.DeClerck, D.Schneider, S.Cartinhour, D.Ware and L.Stein (2002) Development and mapping of 2240 new SSR markers for rice (Oryza sativa L.). DNA Res. 9: 199-207.

Murray,M.G. and W.F.Thompson (1980) Rapid isolation of highmolecular-weight plant DNA. Nucleic Acids Res. 8: 4321-4325.

Nelson,J.C. (1997) QGene: software for marker-based genomic analysis and breeding. Mol. Breed. 3: 239-245.

Oohata,K. (1989) Sheath blight disease. In "Rice Diseases in Japan" Zenkoku Noson Kyoiku Kyokai, Tokyo. p. 409-423.

Pan,X.B., M.C.Rush, X.Y.Sha, Q.J.Xie, S.D.Linscomb, S.R.Stetina and J.H.Oard (1999) Major gene, nonallelic sheath blight resistance from the rice cultivars Jasmin 85 and Teqin. Crop Sci. 39: 338-346.

Temnykh,S., W.D.Park, N.Ayres, S.Cartinhour, N.Hauck, L.Lipovich, Y.G.Cho, T.Ishii and S.R.McCouch (2000) Mapping and genome organization of microsatellite sequences in rice (Oryza sativa L.). Theor. Appl. Genet. 100: 697-712.

Temnykh,S., G.DeClerck, A.Lukashova, L.Lipovich, S.Cartinhour and S.McCouch (2001) Computational and experimental analysis of microsatellites in rice (Oryza sativa L.): Frequency, length variation, transposon associations, and genetic marker potential. Genome Res. 11: 1441-1452.

Wasano,K. (1988) The resistance to the rice diseases of bacterial leaf blight (Xanthomonas campestris pv. Oryzae) and sheath blight (Rhizoctonia solani), and some problems in breeding of disease resistance of crops. In "Recent Advances in Research on Plant Breeding vol. 30" Japanese Society of Breeding (ed.), Yokendo, Tokyo. p. 103-121.

Wasano,K., S.Oro and Y.Kido (1983) The syringe inoculation method for selecting rice plants resistant to sheath blight, Rhizoctonia solani Kuhn. J. Trop. Agr. 27: 131-139.

Wu,J., T.Maehara, T.Shimokawa, S.Yamamoto, C.Harada, Y.Takazaki, N.Ono, Y.Mukai, K. Koike, J.Yazaki, F.Fujii, A.Shomura, T.Ando, I.Kono, K.Waki, K.Yamamoto, M.Yano, T.Matsumoto and T.Sasaki (2002) A comprehensive rice transcript map containing 6591 EST site. Plant Cell 14: 525-535.

Xie, Q.J., M.C.Rush and J.Cao (1990) Somaclonal variation for disease resistance in rice (Oryza sativa L.). In "Pest Management in Rice" Grayson,B.T. and M.B.Green (eds.), Elsevier Applied Sciense, New York. p. 491-509.

Zhang, Q.C., K.P.Che, Z.P.Wang, D.Y.He, D.M.Jin and B.Wang (2001) Novel rice variety development for sheath blight resistance by transferring nuclear DNA of Zizania caduciflora into rice cultivar. Chinese Sci. Abstr. 7: 665.

Zou,J.H., X.B.Pan, Z.X.Chen, J.Y.Xu, J.F.Lu, W.X.Zhai and L.H.Zhu (2000) Mapping quantitative trait loci controlling sheath blight resistance in two rice cultivars (Oryza sativa L.). Theor. Appl. Genet. 101: 569-573. 